\title{
Local Volume Depletion/Accumulation in GAA Si Nanowire Junctionless nMOSFETs
}

\author{
Mohammad Najmzadeh, Student Member, IEEE, Jean-Michel Sallese, Matthieu Berthomé, Student Member, IEEE, \\ Wladek Grabinski, Senior Member, IEEE, and Adrian M. Ionescu, Senior Member, IEEE
}

\begin{abstract}
In this paper, we report, for the first time, corner effect analysis in the gate-all-around equilateral triangular silicon nanowire (NW) junctionless (JL) nMOSFETs, from subthreshold to strong accumulation regime. Corners were found to accumulate and deplete more electrons than the flat sides or the channel center, when above (local accumulation) and below (local depletion) the flat-band voltage, respectively. On the contrary to the corner effect in the inversion mode (IM) devices, there is no major contribution of corners in the subthreshold current, and therefore, there is no subthreshold device behavior degradation (only one threshold voltage in the system). $\mathrm{N}$-type channel doping levels of $1 \times 10^{19}, 5 \times 10^{18}$, and $1 \times 10^{18} \mathrm{~cm}^{-3}$ were used for quasi-stationary device simulations of JL and AM MOSFETs, and corner effect was studied for 5,10 , and $15 \mathrm{~nm}$ wide equilateral triangular $\mathrm{Si} \mathrm{NW}$ MOSFETs with a $2 \mathrm{~nm} \mathrm{SiO}$ gate oxide thickness $\left(V_{\mathrm{DS}}=0 \mathrm{~V} ; T=300 \mathrm{~K}\right)$. While the local quantum and classical electron density peaks are located in the corner regions above the flat-band voltage, reducing the channel doping and the channel cross-section was found to slightly suppress the normalized total accumulation electron density per unit length, $N_{t}^{\text {acc }} /\left(\mathrm{CW}_{\text {eff }}\right)$, in strong accumulation regime.
\end{abstract}

Index Terms-Accumulation mode (AM), corner effect, gateall-around (GAA), junctionless (JL), local accumulation, local depletion, quantum confinement, Si nanowire (NW), 3-D TCAD Sentaurus Device simulation.

\section{INTRODUCTION}

M ULTI-GATE architectures such as gate-all-around nanowires and FinFETs are promising candidates for aggressive CMOS downscaling, due to an almost optimized subthreshold slope, immunity against short channel effects, and optimized power consumption. Recently, highly and heavily single-type doped Si devices along the source-channel-drain, called accumulation mode (AM) and junctionless (JL), have been proposed [1], [2]. These devices present a simpler fabrication method to overcome some technical limitations of junction-based devices like ultra-abrupt junctions, which are

Manuscript received May 23, 2012; revised August 7, 2012; accepted September 7, 2012. Date of publication October 19, 2012; date of current version November 16, 2012. This work was supported by the Swiss National Science Foundation. The review of this paper was arranged by Editor K. Roy.

M. Najmzadeh, M. Berthomé, W. Grabinski, and A. M. Ionescu are with the Nanoelectronic Devices Laboratory, Swiss Federal Institute of Technology in Lausanne (EPFL), 1015 Lausanne, Switzerland (e-mail: mohammad.najmzadeh@epfl.ch; matthieu.berthome@epfl.ch; wladyslaw.grabinski@epfl.ch; adrian.ionescu@epfl.ch).

J.-M. Sallese is with the STI Scientists Group, Swiss Federal Institute of Technology in Lausanne (EPFL), 1015 Lausanne, Switzerland (e-mail: jean-michel.sallese@epfl.ch).

Color versions of one or more of the figures in this paper are available online at http://ieeexplore.ieee.org.

Digital Object Identifier 10.1109/TED.2012.2220363 issues for ultra short channel devices. The multi-gate architectures (except circular cross-sections that can be obtained by hydrogen annealing [3] or stress-limited oxidation [4]) have corners (e.g., see [5]-[9]). Therefore, an in-depth analysis of the corner effect on the electrical characteristics of the multigate devices is necessary. In this paper, we report, for the first time, corner effect analysis of the GAA Si NW JL nMOSFETs using a GAA equilateral triangular Si NW architecture $\left(2 \mathrm{~nm} \mathrm{SiO}_{2}\right.$ gate oxide thickness; $\left.V_{\mathrm{DS}}=0 \mathrm{~V} ; T=300 \mathrm{~K}\right)$. The corner effect analysis was done from subthreshold to strong accumulation, considering various channel doping levels $(1 \times$ $10^{18}-1 \times 10^{19} \mathrm{~cm}^{-3}$ ) and channel cross-sectional dimensions (5-15 nm Si NW width).

To make a clear corner effect study in GAA Si NW JL MOSFETs with minimized short channel effects on the device characteristics, $40 \mathrm{~nm}$ long channel architectures were used for the simulations $(>6$ times longer than the natural length of the widest NW; see, e.g., [10]). This is a first step to make a precise device and transport analysis in multi-gate JL architectures with short channel lengths including corners (see e.g., [11]). Note that various Si NW cross-sections can be experimentally achievable using bottom-up [12], [13] or Si NW sidewall engineering by anisotropic Si etching in topdown [6], [14] platforms. In this paper, we only concentrate on the equilateral triangular cross-sections, due to having the narrowest corner angle among the symmetrical architectures.

In this paper and as a first step, we investigate the corner effect through the local and total electron densities in the channel cross-section (with and without channel quantization) using a $15 \mathrm{~nm}$ wide Si NW MOSFET at various channel doping levels. Afterward, the effect of channel dimension shrinkage in a JL MOSFET at a fixed channel doping level will be studied in details.

\section{Numerical Simulation}

TCAD Sentaurus Device (G-2012.06) was used for the quasistationary numerical simulation of GAA Si NW MOSFETs. Considering electrostatic and quasi-Fermi potential equations, the local carrier densities in a 3-D structure can be extracted at each bias voltage. The electrostatic potential for the classic case is the solution of the nonlinear Poisson equation

$$
\nabla \cdot(\epsilon \nabla \psi)=-q\left(-n+p+N_{D}^{+}\right)
$$

where $q, n, p$, and $N_{D}^{+}$are electron charge, electron density, hole density, and ionized donor concentration, respectively 

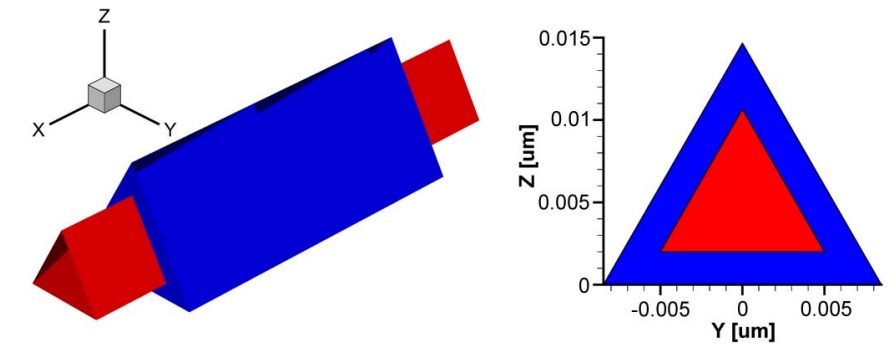

Fig. 1. Equilateral triangular GAA Si NW MOSFET and its cross-section.

(ionized acceptor concentration is neglected in our case). To include the 3-D quantization effects in nanoscale, the density gradient quantization model is coupled to the Poisson equation [15], [16]. The quantum correction procedure includes modification of the density of states [16]. The semi-classical Slotboom bandgap narrowing model was used for the highly and heavily doped Si channels [16], [17]. The local carrier densities can be computed from the electron and hole quasiFermi potentials, considering Fermi-Dirac statistics covering both degenerate and nondegenerate regimes [16].

Fig. 1 shows the 3-D GAA Si NW architecture, used for the device simulations (gate length: $40 \mathrm{~nm} ; \mathrm{SiO}_{2}$ gate oxide thickness: $2 \mathrm{~nm}$ ). The Si NW width is set to $15 \mathrm{~nm}$ (equilateral triangle), and three channel doping levels were investigated. The gate workfunction was set to $4.5 \mathrm{eV}$ (a midgap workfunction). In all the simulations, $V_{\mathrm{DS}}$ was fixed at $0 \mathrm{~V}$, to eliminate the effect of longitudinal electric field from source-drain potential difference on the local electron density distribution along the channel.

\section{From Subthreshold to Strong ACcumulation IN A $15 \mathrm{~nm}$ WIDE Si NW MOSFET}

Quasi-stationary TCAD device simulation was done on a $15 \mathrm{~nm}$ wide Si NW MOSFET at three channel doping levels $\left(1 \times 10^{19}, 5 \times 10^{18}\right.$, and $\left.1 \times 10^{18} \mathrm{~cm}^{-3}\right)$. The device characteristics can be studied using local electron density distribution in the channel at each gate voltage as well as charge on the gate versus gate voltage characteristics $\left(Q_{G}-V_{\mathrm{GS}}\right.$, can be obtained directly from the simulations).

\section{A. Operation of AM/JL MOSFETS}

The JL and AM MOSFETs, unlike the typical inversionmode (IM) MOSFETs, do not have any p-n junction, and the channel doping level is nominally determining the device type (heavily doped devices, $>1 \times 10^{19} \mathrm{~cm}^{-3}$, called JL) [1]. Both devices have the same operation mechanism, while here, we provide a brief explanation on this mechanism for a simple planar single-gate AM/JL nMOSFET.

Below the threshold voltage, the channel is fully depleted, while the majority of the subthreshold current is passing through the channel volume (the closer to the channeldielectric interface, the more depletion). There can be different definitions of threshold voltage. Whereas a simple extrapolation was proposed in [18], in this paper, we will adopt a slightly different condition. Assuming a full depletion approximation, our

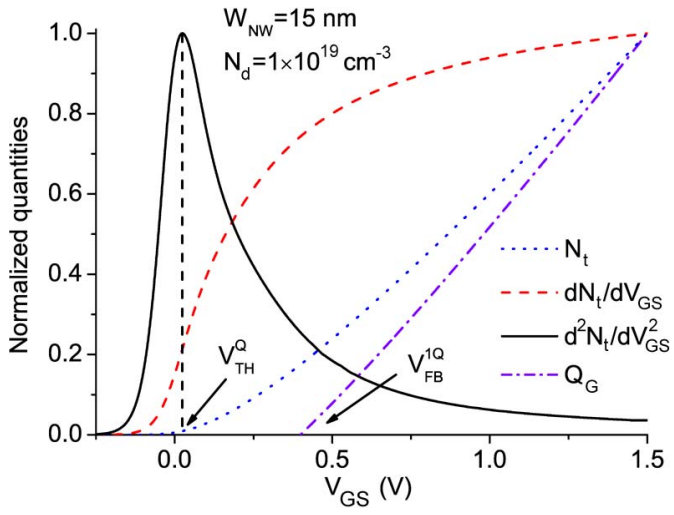

Fig. 2. Normalized quantities of $N_{t}, d N_{t} / d V_{\mathrm{GS}}, d^{2} N_{t} / d V_{\mathrm{GS}}^{2}$, and $Q_{G}$ with respect to the maximum values versus $V_{\mathrm{GS}}$ for the GAA $15 \mathrm{~nm}$ wide Si NW MOSFET at $1 \times 10^{19} \mathrm{~cm}^{-3}$ channel doping including quantum confinement. The maximum values for each parameter are $5.46 \times 10^{7} \mathrm{~cm}^{-1}$, $4.48 \times 10^{7} \mathrm{~cm}^{-1} \cdot \mathrm{V}^{-1}, 1.29 \times 10^{8} \mathrm{~cm}^{-1} \cdot \mathrm{V}^{-2}$, and $7.35 \times 10^{-12} \mathrm{C} \cdot \mathrm{cm}^{-1}$, respectively.

threshold voltage condition can be approximated when creating a neutral region at the middle of the fully depleted channel (the corresponding local electron density almost equals the channel doping). The current passing through the neutral region is called bulk current. Applying a higher gate voltage extends the neutral region causing an increase in the bulk current.

The flat-band condition will be reached when the entire channel cross-section is neutral, implying that the bulk current will saturate at this point. Applying a higher gate voltage leads to the creation of an accumulation layer close to the channeldielectric interface. Therefore, the drain current includes one fixed (saturated bulk current) and one variable (accumulation current) component. Note that the $V_{\mathrm{FB}}-V_{\mathrm{TH}}$ can be engineered by channel doping, gate oxide thickness, channel crosssectional geometry, and dimension.

\section{B. Threshold Voltage Extraction Method}

The threshold voltage can be extracted from the peak of the second derivative of the total electron density per unit length $\left(N_{t}\right)$ versus gate voltage [19] (similar to the transconductance change method [20]), while $N_{t}$ can be calculated by integrating the electron density over the channel cross-section and at the middle of the channel ( $x=L_{G} / 2 ; L_{G}$ equals gate length)

$$
N_{t}=\iint n(y, z) d y d z \text {. }
$$

Fig. 2 shows the total electron density per unit length and the corresponding derivatives (normalized to the corresponding maximum values) for a GAA $15 \mathrm{~nm}$ wide Si NW MOSFET doped at $1 \times 10^{19} \mathrm{~cm}^{-3}$, including quantum confinement.

\section{Flat-band Voltage Extraction Method}

In a standard planar MOSFET, the flat-band voltage is the gate voltage for which the electrostatic potential is being constant in the entire channel cross-section. However, due to the quantum confinement, the flat-band condition cannot be reached in the entire channel cross-section for a certain gate 
TABLE I

Key Device Parameter Extraction From the Quasi-Static Device Simulations of THE GAA $15 \mathrm{~nm}$ Wide Si NW MOSFETs at DifFERENT ChanNel Doping LEVEls

\begin{tabular}{c|c|c|c|c|c|c|c|c}
\hline $\begin{array}{c}\mathrm{N}_{d} \\
\left(\mathrm{~cm}^{-3}\right)\end{array}$ & $\begin{array}{c}\mathrm{V}_{T H}^{C} \\
(\mathrm{~V})\end{array}$ & $\begin{array}{c}\mathrm{V}_{T H}^{Q} \\
(\mathrm{~V})\end{array}$ & $\begin{array}{c}\mathrm{V}_{F B}^{1 C} \\
(\mathrm{~V})\end{array}$ & $\begin{array}{c}\mathrm{V}_{F B}^{1 Q} \\
(\mathrm{~V})\end{array}$ & $\begin{array}{c}\mathrm{V}_{F B}^{C} \\
(\mathrm{~V})\end{array}$ & $\begin{array}{c}\mathrm{V}_{F B}^{Q} \\
(\mathrm{~V})\end{array}$ & $\begin{array}{c}\mathrm{CW}_{\text {eff }}^{\text {Cmax }} \\
(\mathrm{F} / \mathrm{cm})\end{array}$ & $\begin{array}{c}\mathrm{CW}_{\text {eff }}^{Q \max } \\
(\mathrm{F} / \mathrm{cm})\end{array}$ \\
\hline $1 \times 10^{18}$ & 0.306 & 0.316 & 0.332 & 0.351 & 0.319 & 0.338 & $7.62 \times 10^{-12}$ & $7.11 \times 10^{-12}$ \\
\hline $5 \times 10^{18}$ & 0.181 & 0.183 & 0.349 & 0.379 & 0.345 & 0.375 & $7.63 \times 10^{-12}$ & $7.14 \times 10^{-12}$ \\
\hline $1 \times 10^{19}$ & 0.025 & 0.027 & 0.360 & 0.399 & 0.360 & 0.399 & $7.65 \times 10^{-12}$ & $7.16 \times 10^{-12}$ \\
\hline
\end{tabular}

voltage when including corners. Nevertheless, we can still define an effective flat-band voltage for the entire channel crosssection (or quantum flat-band voltage) as a key device operation parameter which can be approximated from the $x$-intercept of the $Q_{G}-V_{\mathrm{GS}}$ curve as a first step (called $V_{\mathrm{FB}}^{1 Q}$ ), a direct output result from the presented gate charge- $V_{\mathrm{GS}}$ quasi-static simulations.

While the flat-band condition can be reached in the entire channel cross-section when discarding quantization, even in the corners, the observed slight difference between the actual flatband voltage $\left(V_{\mathrm{FB}}^{C}\right)$ and the extracted one from the $Q_{G}-V_{\mathrm{GS}}$ curve for the classic case, $V_{\mathrm{FB}}^{1 C}-V_{\mathrm{FB}}^{C}$, can be used to justify the flat-band voltage extraction method mismatch when quantum effects cannot be neglected. This slight inaccuracy, observed to be below a $13 \mathrm{mV}$ range in Table $\mathrm{I}$, is mainly due to the higher electron density in the channel parts close to the source and drain, as well as to the parameter extraction methodology. Based on this remark, we can estimate the effective flat-band voltage for the entire device $\left(V_{\mathrm{FB}}^{Q}\right.$ or $\left.V_{\mathrm{FB}}^{C}\right)$.

Note that the flat-band condition may not be reached in the entire channel cross-section even for the classic case. This could be due to some local effective bulk doping concentrations in the corners, as reported previously in the subthreshold regime of the IM devices to describe the local threshold voltage downshift in the corners [19], [21]. However, perhaps a much narrower corner angle is needed to significantly affect the local flat-band voltage variation between the corner and the side.

\section{Gate-Channel Capacitance and Effective Channel Width}

Due to the quantization-based gate-channel capacitance [22] and effective channel width shrinkages in GAA NWs, instead of extracting each parameter separately, the $\mathrm{CW}_{\text {eff }}$ parameter, the product of the gate-channel capacitance and the channel width, is introduced. This parameter can be extracted from the first derivative of the total electron density per unit length $\left(N_{t}\right)$ versus the gate voltage in strong accumulation regime

$$
\mathrm{CW}_{\mathrm{eff}}\left(V_{\mathrm{GS}}\right)=\left(d N_{t} / d V_{\mathrm{GS}}\right) \cdot q .
$$

The $\mathrm{CW}_{\mathrm{eff}}^{\max }$ values, reported in Table I, are extracted at $V_{\mathrm{GS}}=$ $1.500 \mathrm{~V}$ for all structures.

\section{E. Key MOSFET Parameters at Different Channel Doping Levels}

Fig. 3 shows the second derivative of the total electron density per unit length $\left(d^{2} N_{t} / d V_{\mathrm{GS}}^{2}\right)$ versus $V_{\mathrm{GS}}$ for the GAA

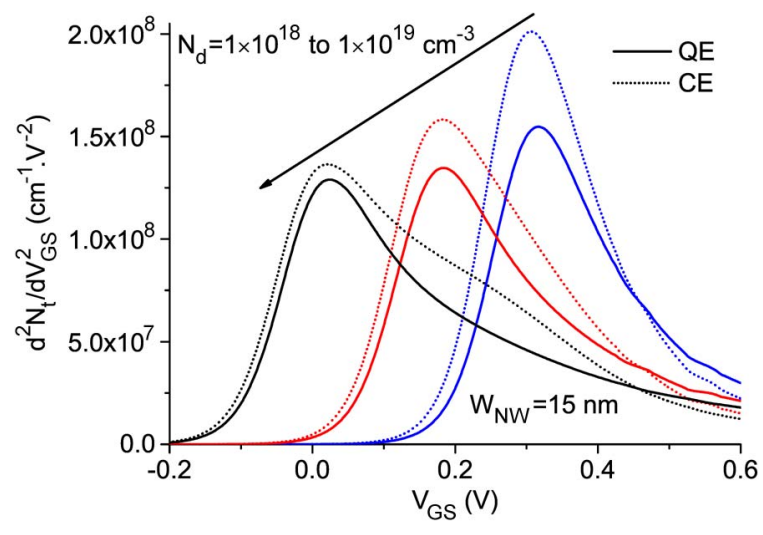

Fig. 3. $d^{2} N_{t} / d V_{\text {GS }}^{2}$ versus gate voltage for the GAA Si NW MOSFETs at various doping levels with or without quantization (QE or CE, respectively).

$15 \mathrm{~nm}$ wide Si NW MOSFETs for three channel doping concentrations, considering classical and quantum effects. The results reported in Table I show that the quantization is upshifting both the threshold and the flat-band voltages, due to the higher quantized subband energies [9], [23], [24]. Note that, even for the heavily doped structure and on the contrary to the IM devices [19], there is no hump effect below the gate voltage corresponding to the main peak in the $d^{2} N_{t} / d V_{\mathrm{GS}}^{2}$ versus $V_{\mathrm{GS}}$ curve, thus representing a unique threshold voltage in the system. The hump appearing above the threshold voltage of the heavily doped device in the classical simulation is due to the nonlinear operation of bulk regime between the threshold and the flat-band voltages as well as creation of accumulation conduction paths in the channel, reported before for the planar AM devices [2].

\section{Local Electron Density Distribution Across THE CHANNEl From SubThreshold TO STRONG ACCUMULATION}

Figs. 4 and 5 show the quantum electron density (QED) and classical electron density (CED) in the cross-section of a GAA $15 \mathrm{~nm}$ wide Si NW JL MOSFET (channel doping: $1 \times 10^{19} \mathrm{~cm}^{-3}$ ) in subthreshold, above threshold, and strong accumulation regimes. According to the figures, the majority of electrons are accommodated in the corner regions only in strong accumulation. To study better the bias-dependent charge distribution mechanism in the channel cross-section, local QED and CED profiles as functions of gate voltage are plotted along $y=0$ (see e.g., Fig. 4) in Fig. 6. This provides a wide range of information on the local electron density variation in the corner, side, and volume.

The maximum and minimum of the local CEDs in accumulation and depletion regimes are both occurring on the $\mathrm{Si}$ NW-dielectric interface, respectively. Therefore, a simple way to study the effect of corners on the local electron density variation can be the local CED corner to side ratio at different channel doping and gate voltages. Note that, due to the quantization effects, the peak of QED occurs inside the channel volume. Fig. 6 inset shows this classical ratio as a function of $V_{\mathrm{GS}}-V_{\mathrm{FB}}$. According to this figure (as well as from the local CEDs at different channel doping levels in Fig. 7), 

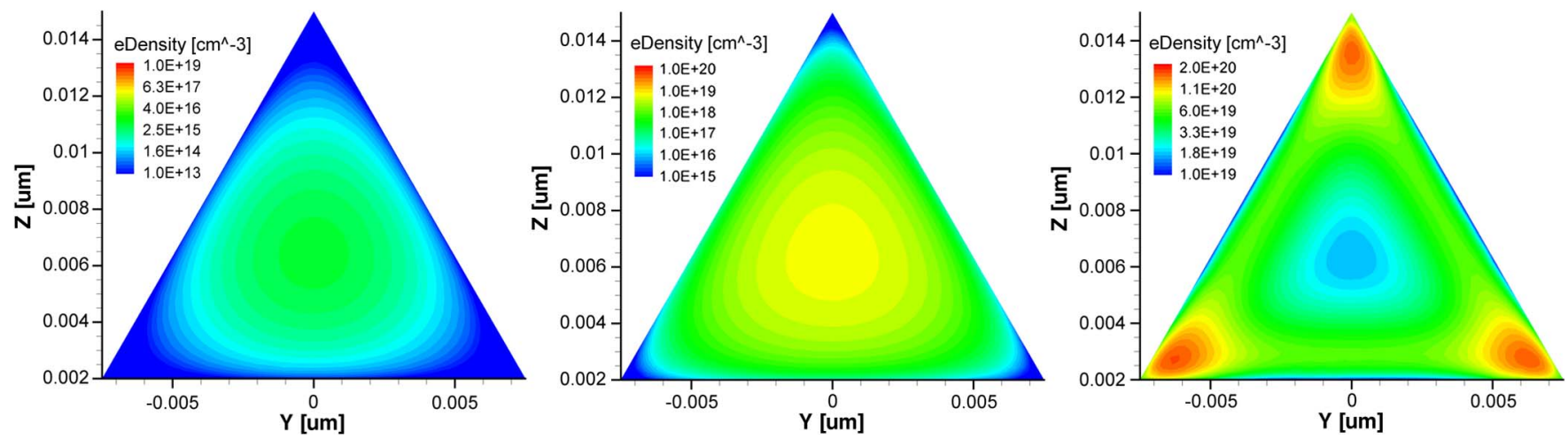

Fig. 4. Cross-sectional QED at the middle of a GAA $15 \mathrm{~nm}$ wide Si NW JL MOSFET for three operation regimes (oxide is not shown; channel doping: $\left.1 \times 10^{19} \mathrm{~cm}^{-3}\right)$. (Left) Subthreshold $\left(V_{\mathrm{GS}}=-0.200 \mathrm{~V}\right)$. (Center) Above threshold $\left(V_{\mathrm{GS}}=0.100 \mathrm{~V}\right)$. (Right) Strong accumulation $\left(V_{\mathrm{GS}}=1.500 \mathrm{~V}\right)$. Note that $V_{\mathrm{TH}}^{Q}=0.027 \mathrm{~V}$ and $V_{\mathrm{FB}}^{Q}=0.399 \mathrm{~V}$.
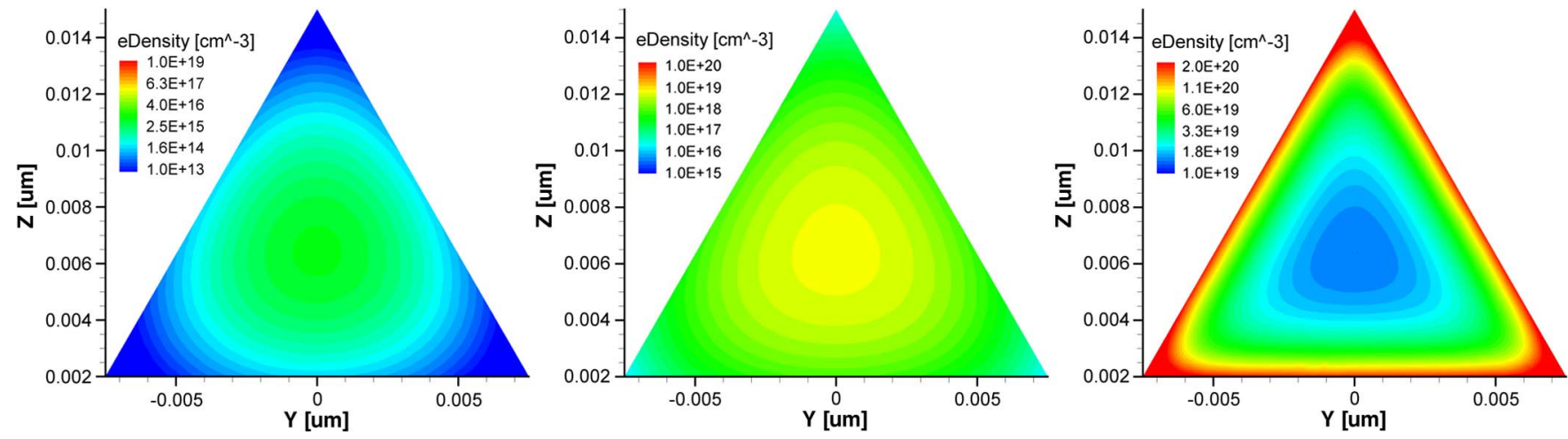

Fig. 5. Cross-sectional CED at the middle of a GAA $15 \mathrm{~nm}$ wide Si NW JL MOSFET for three operation regimes (oxide is not shown; channel doping: $\left.1 \times 10^{19} \mathrm{~cm}^{-3}\right)$. (Left) Subthreshold $\left(V_{\mathrm{GS}}=-0.200 \mathrm{~V}\right)$. (Center) Above threshold $\left(V_{\mathrm{GS}}=0.100 \mathrm{~V}\right)$. (Right) Strong accumulation $\left(V_{\mathrm{GS}}=1.500 \mathrm{~V}\right)$. Note that $V_{\mathrm{TH}}^{C}=0.025 \mathrm{~V}$ and $V_{\mathrm{FB}}^{C}=0.360 \mathrm{~V}$.

corners accumulate more electrons in comparison to the side in accumulation regime $\left(>V_{\mathrm{FB}}\right)$, while they deplete also further below the flat-band voltage.

\section{A. Origin of Local Depletion/Local Accumulation in AM/JL MOSFETS}

The different corner effects and device behavior in the IM and the AM/JL MOSFETs come from a distinct conduction mechanism, surface versus volume conduction in different regimes, as well as conduction of minority versus majority carriers. Analyzing the effect of corners on the carrier density distribution in the channel cross-section is not simple. According to the simulations, it strongly depends on the channel geometry, doping level, and dielectric thickness (see e.g., [19], [25]-[27]). There is no clear geometrical definition of the corner region, while the analysis becomes even more complex including quantization.

The surface conduction by minority carriers is the only conduction mechanism in the IM devices, while the AM/JL MOSFETs exhibit surface conduction above $V_{\mathrm{FB}}$ and volume conduction below $V_{\mathrm{FB}}$, both involving majority carriers. Due to having a maximized surface to volume ratio in the corner region in comparison to the side region, the surface conduction mechanisms (above $V_{\mathrm{FB}}$ for the $\mathrm{AM} / \mathrm{JL}$, all operation regimes for the IM devices) should provide a higher local mobile charge density in the corner region (local volume inversion or local volume accumulation in the IM and the AM/JL devices, respectively). On the other hand, reduction of the local effective channel doping in the corners because of side gates and the effective body thickness reduction in the corners were suggested previously to describe the local threshold voltage downshift and the local volume inversion in the corners of the IM devices in subthreshold regime as well [21].

Due to having a smaller effective channel body thickness in the corner region in comparison to the side, the volume conduction mechanism in the corner region is expected to be minimized with respect to the side region below the flat-band voltage, since the volume of corner is negligible. Therefore, no subthreshold conduction path in the corner regions of the AM/JL MOSFETs is expected to emerge from $I_{D}-V_{\mathrm{GS}}$ characteristics, as already observed in Fig. 3 (no hump below the main peak of the $d^{2} N_{t} / d V_{\mathrm{GS}}^{2}$ versus $V_{\mathrm{GS}}$ curves).

\section{B. Corner Effects on Global Accumulation Electron Densities in Accumulation Regime}

To assess corner effects on the global device characteristics, the normalized total accumulation electron density per unit length in the entire channel cross-section is defined as $\left(V_{\mathrm{GS}}>V_{\mathrm{FB}}\right)$

$$
N_{t}^{\mathrm{acc}}\left(V_{\mathrm{GS}}\right)=N_{t}\left(V_{\mathrm{GS}}\right)-N_{t}\left(V_{\mathrm{FB}}\right) .
$$



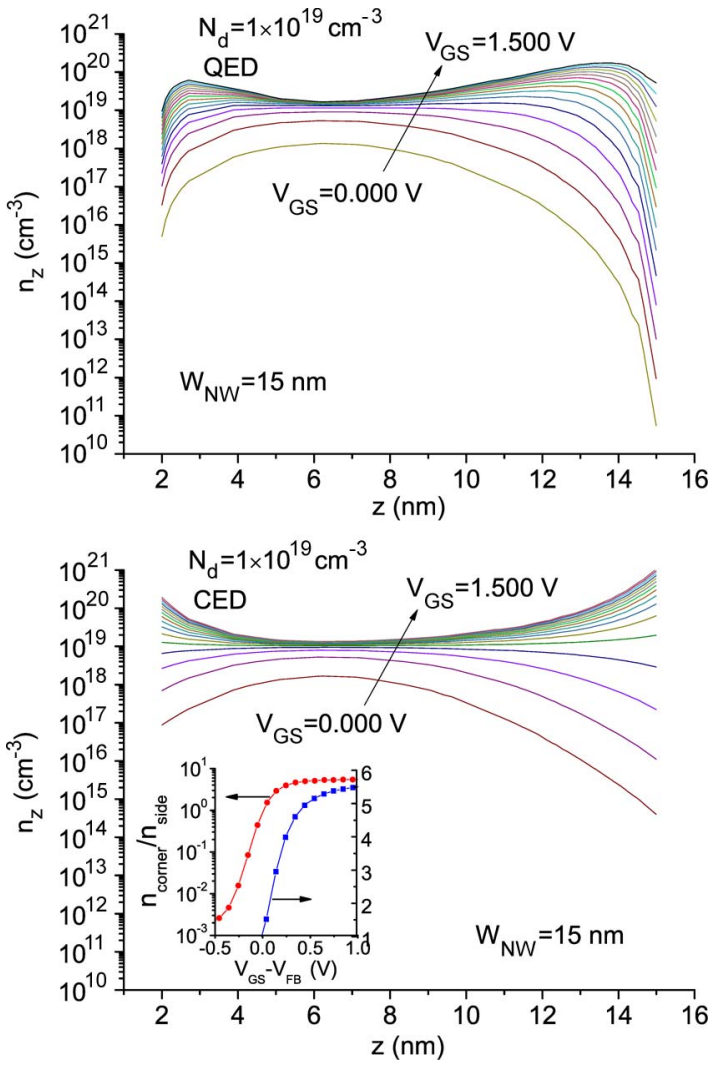

Fig. 6. Local (top) QED and (bottom) CED profiles across the $15 \mathrm{~nm}$ wide NW channel volume at different $V_{\mathrm{GS}}$ values (from subthreshold to strong accumulation; step: $0.100 \mathrm{~V}$ ) at $N_{d}=1 \times 10^{19} \mathrm{~cm}^{-3}$ (cut at $y=0$; see e.g., Fig. 4). The inset shows local CED corner to side ratio from subthreshold to strong accumulation.

Fig. 8 shows how this normalized accumulation electron density varies with respect to the gate voltage for the $15 \mathrm{~nm}$ wide Si NW MOSFETs, with three different channel doping levels at $V_{\mathrm{GS}}>V_{\mathrm{FB}}$. In order to study the effect of quantization and channel cross-sectional variation for various devices, accumulation electron densities are normalized to $\mathrm{CW}_{\text {eff }}\left(V_{\mathrm{GS}}\right)$ at each bias voltage (see also Section V-A). According to Fig. 8, the normalized total accumulation electron density above the flatband voltage is increasing with the channel doping, while all the normalized values are slightly below the ideal limit that can be calculated as follows:

$$
N_{t}^{\mathrm{acc}}\left(V_{\mathrm{GS}}\right) /\left[\mathrm{CW}_{\mathrm{eff}}\left(V_{\mathrm{GS}}\right)\right]=\left(V_{\mathrm{GS}}-V_{\mathrm{FB}}\right) / q \text {. }
$$

Therefore, corners clearly cannot be considered as CMOS boosters. Note that the local CED corner to side ratio is increasing by channel doping reduction in accumulation regime (Fig. 8 inset). On the other hand and from Fig. 8, heavily doped structures represent a higher normalized total accumulation electron density per unit length and reveal characteristics closer to the ideal case, reflecting a more uniform distribution of the local electrons in the cross-section. This could be explained by electrostatic screening increase at higher doping levels in accumulation regime. In all cases, the normalized total accumulation electron density per unit length is slightly degraded by quantum confinement as well.
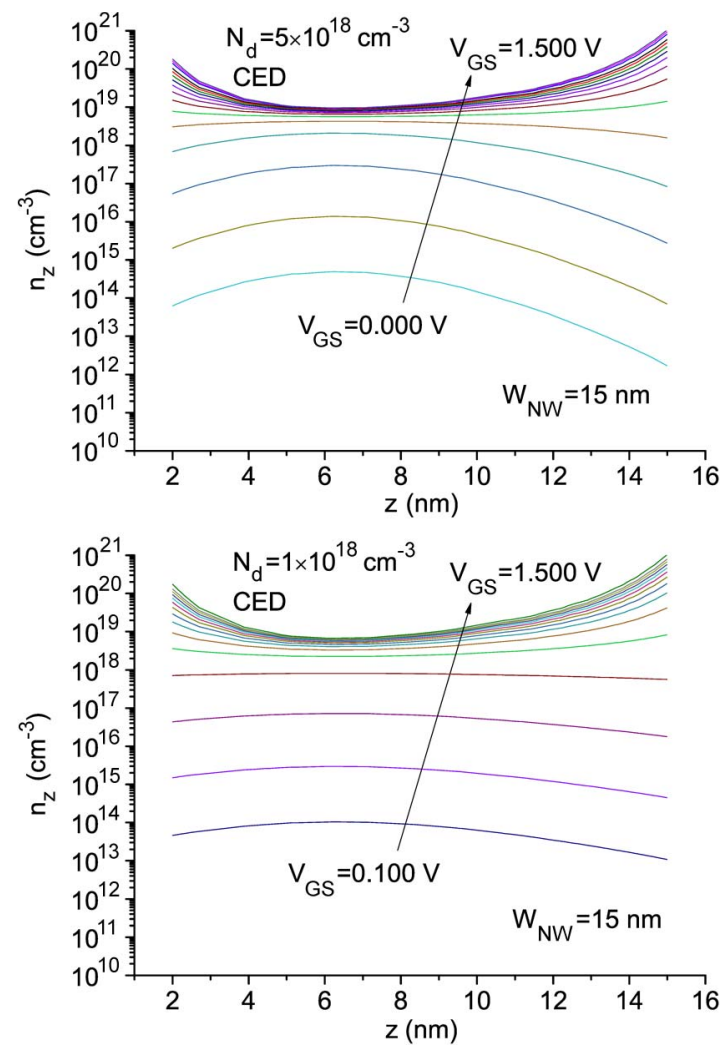

Fig. 7. Local CED profiles across the $15 \mathrm{~nm}$ wide Si NW channel volume at different gate voltages (from subthreshold to strong accumulation; step: $0.100 \mathrm{~V}$ ) for (top) $N_{d}=5 \times 10^{18} \mathrm{~cm}^{-3}$ and (bottom) $1 \times 10^{18} \mathrm{~cm}^{-3}$. The plots correspond to the cut at $y=0$.

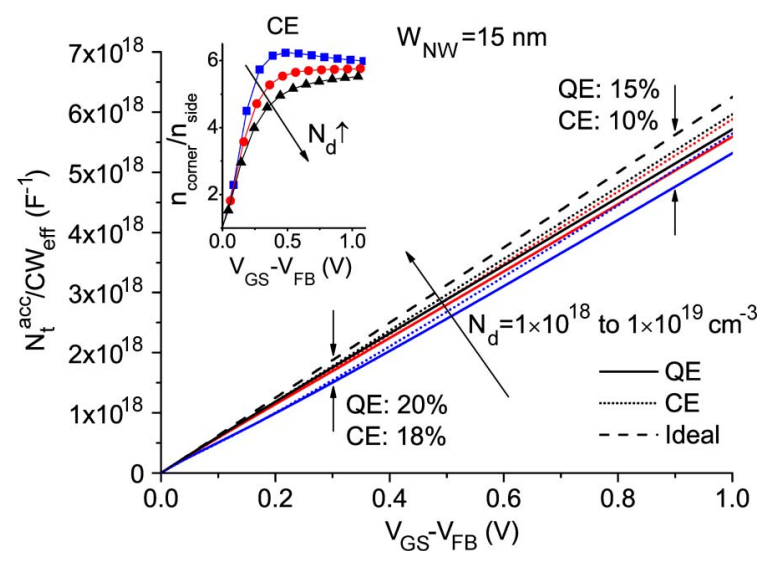

Fig. 8. Normalized total accumulation electron density per unit length versus $V_{\mathrm{GS}}-V_{\mathrm{FB}}$ at various channel doping levels including both quantum and classical electrons. The normalization factor is $\mathrm{CW}_{\mathrm{eff}}\left(V_{\mathrm{GS}}\right)$. The inset shows local CED corner to side ratios in accumulation regime.

\section{Cross-Sectional Shrinkage And Corner EFfect}

In this section, GAA equilateral triangular $\mathrm{Si} \mathrm{NW}$ MOSFETs with 5 and $10 \mathrm{~nm}$ NW widths were simulated at a $1 \times 10^{19} \mathrm{~cm}^{-3}$ channel doping level $\left(2 \mathrm{~nm} \mathrm{SiO}_{2}\right.$ gate oxide thickness). The second derivative of $N_{t}\left(d^{2} N_{t} / d V_{\mathrm{GS}}^{2}\right)$ versus gate voltage curves are plotted in Fig. 9, and the extracted device parameters are reported in Table II (the 


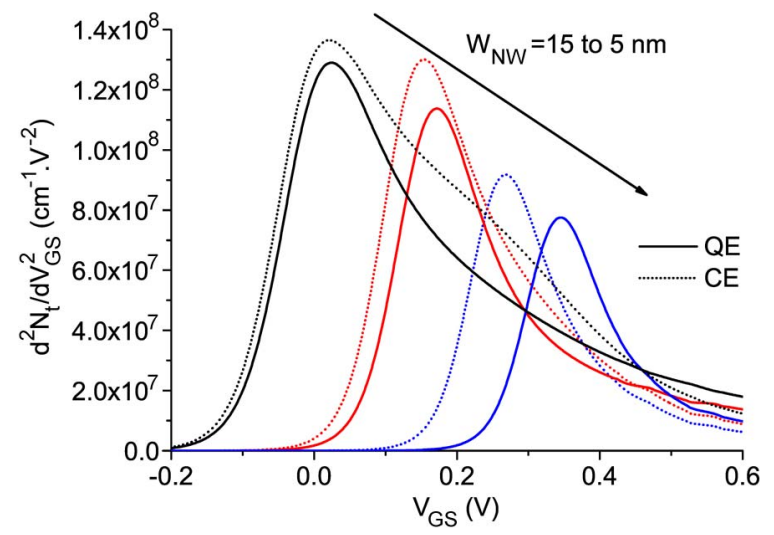

Fig. 9. $d^{2} N_{t} / d V_{\text {GS }}^{2}$ versus gate voltage for the GAA Si NW MOSFETs for various NW widths doped at $N_{d}=1 \times 10^{19} \mathrm{~cm}^{-3}$, including both (QE) quantum and (CE) classical electrons.

TABLE II

Key DeVice Parameter Extraction From the Quasi-STationary DEVICE Simulations OF THE GAA Si NW MOSFETs With VARIOUS NW WidTHS, ALL AT $N_{d}=1 \times 10^{19} \mathrm{~cm}^{-3}$

\begin{tabular}{c|c|c|c|c|c|c|c|c}
\hline $\begin{array}{c}\mathrm{W}_{N W} \\
(\mathrm{~nm})\end{array}$ & $\begin{array}{c}\mathrm{V}_{T H}^{C}(\mathrm{~V}) \\
(\mathrm{V})\end{array}$ & $\begin{array}{c}\mathrm{V}_{T H}^{Q} \\
(\mathrm{~V}) \\
(\mathrm{V})\end{array}$ & $\begin{array}{c}\mathrm{V}_{F B}^{1 Q} \\
(\mathrm{~V})\end{array}$ & $\begin{array}{c}\mathrm{V}_{F B}^{C}(\mathrm{~V}) \\
(\mathrm{V})\end{array}$ & $\begin{array}{c}\mathrm{V}_{F B}^{Q} \\
(\mathrm{~V})\end{array}$ & $\begin{array}{c}\mathrm{CW}_{\text {eff }}^{C \max } \\
(\mathrm{F} / \mathrm{cm})\end{array}$ & $\begin{array}{c}\mathrm{CW}_{\text {eff }}^{Q m a x} \\
(\mathrm{~F} / \mathrm{cm})\end{array}$ \\
\hline 5 & 0.267 & 0.344 & 0.360 & 0.447 & 0.360 & 0.447 & $2.86 \times 10^{-12}$ & $2.66 \times 10^{-12}$ \\
\hline 10 & 0.153 & 0.171 & 0.360 & 0.408 & 0.360 & 0.408 & $5.25 \times 10^{-12}$ & $4.94 \times 10^{-12}$ \\
\hline 15 & 0.025 & 0.027 & 0.360 & 0.399 & 0.360 & 0.399 & $7.65 \times 10^{-12}$ & $7.16 \times 10^{-12}$ \\
\hline
\end{tabular}
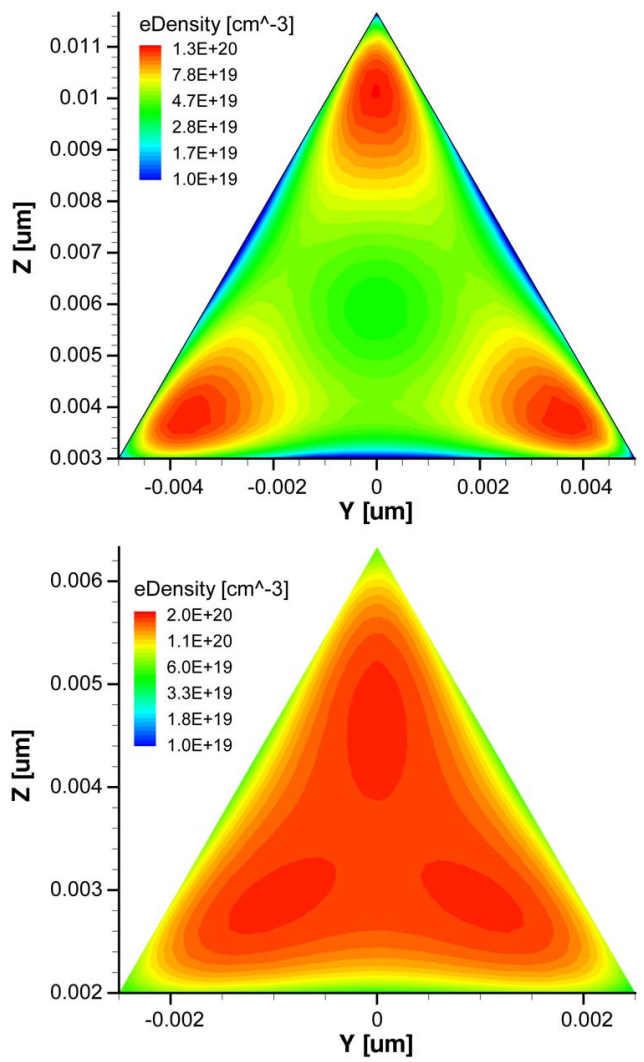

Fig. 10. Cross-sectional QED at the middle of a GAA Si NW JL MOSFET in strong accumulation regime $\left(V_{\mathrm{GS}}=1.500 \mathrm{~V}\right)$ for (top) 10 and (bottom) $5 \mathrm{~nm}$ wide Si NW MOSFETs at $N_{d}=1 \times 10^{19} \mathrm{~cm}^{-3}$.
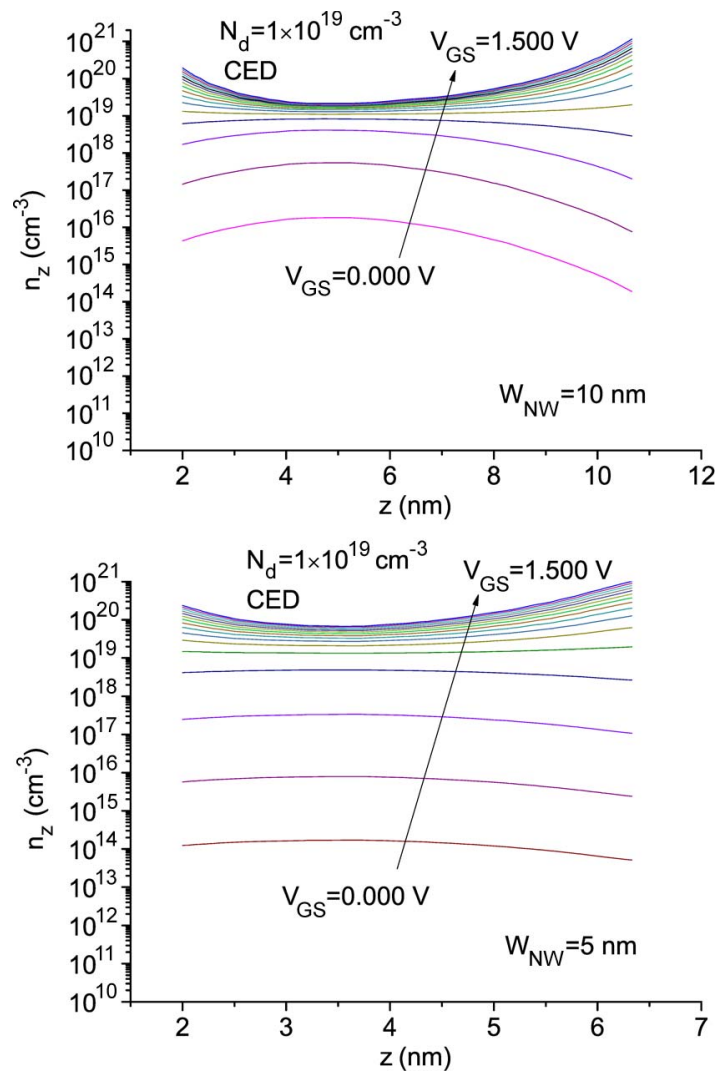

Fig. 11. Local CED profile across the Si NW channel volume at different gate voltages (from subthreshold to strong accumulation; step: $0.100 \mathrm{~V}$ ) for (top) 10 and (bottom) $5 \mathrm{~nm}$ wide Si NW MOSFETs at $N_{d}=1 \times 10^{19} \mathrm{~cm}^{-3}$. Plots correspond to the cut at $y=0$.

$15 \mathrm{~nm}$ wide NW with a similar doping is added from Table I for comparison). No hump exists below the threshold voltage, while the one above the threshold voltage for the classical simulation disappears by cross-sectional shrinkage as well, mainly due to the reduction of bulk conduction regime $\left(V_{\mathrm{FB}}-V_{\mathrm{TH}}\right)$.

\section{A. Local Electron Density for Various Cross-Sectional Dimensions}

Fig. 10 shows the cross-sectional local electron density distribution in the channel with quantization for 10 and $5 \mathrm{~nm}$ wide $\mathrm{Si} \mathrm{NW}$ JL MOSFETs in strong accumulation $\left(V_{\mathrm{GS}}=1.500 \mathrm{~V}\right)$. Significant charge redistribution in the $5 \mathrm{~nm}$ wide NW cross-section, which can be called volume accumulation, in comparison to the wider ones together with 77 and $87 \mathrm{mV}$ upshifts in the threshold and the flat-band voltages, respectively, are the typical quantization effects in such scaled 1DEG architectures. The local CED profiles along $y=0$ are plotted in Fig. 11 for both devices at different $V_{\mathrm{GS}}$ values. According to Fig. 12, the normalized total accumulation electron density per unit length becomes closer to the ideal limit for the wider structures. This corner effect is pretty close to the one occurring in the IM devices, simply due to less contribution of the corner regions on the electrostatics in the entire channel cross-section and less quantization effects for wider structures. 


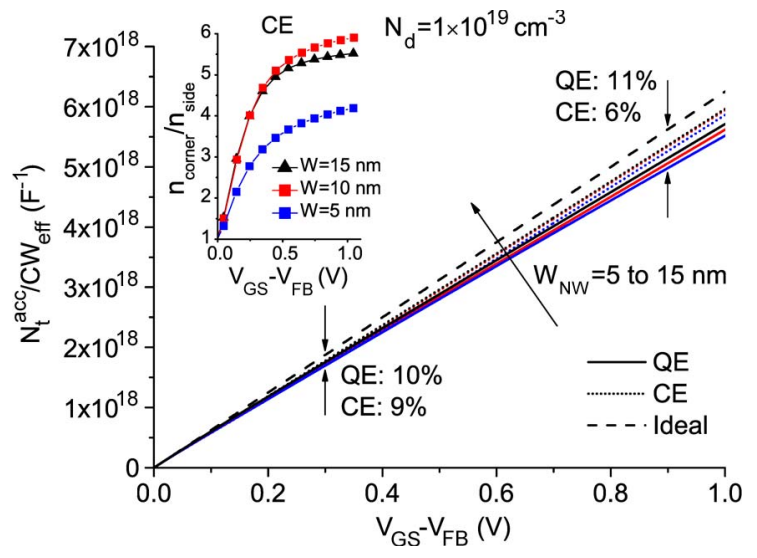

Fig. 12. Normalized total accumulation electron density per unit length versus $V_{\mathrm{GS}}-V_{\mathrm{FB}}$ at various $\mathrm{NW}$ cross-sectional dimensions including both quantum and classical electrons. The normalization factor is $\mathrm{CW}_{\mathrm{eff}}\left(V_{\mathrm{GS}}\right)$. The inset shows local CED corner to side ratios in accumulation regime.

\section{CONCLUSION}

In this paper, we have reported corner effect study in the single-type doped (AM and JL) GAA equilateral triangular Si NW nMOSFETs for the first time. In the IM MOSFETs, the corners slightly degrade the subthreshold behavior due to the parasitic corner conductions below the threshold voltage. Therefore, the typical well-known corner effect (local threshold voltage downshift in the corners and, therefore, increased OFF current) is suppressed completely using AM and JL architectures by having a unique threshold voltage in the system. On the other hand, the corners cannot be classified as CMOS boosters (e.g., stressors), due to having a normalized total accumulation electron density per unit length [normalization factor: $\mathrm{CW}_{\text {eff }}\left(V_{\mathrm{GS}}\right)$ ] slightly below the ideal MOSFET limit, even having a higher local charge accumulation in the corner regions in comparison to the side regions above flat-band.

\section{ACKNOWLEDGMENT}

The authors would like to thank Prof. A. Schenk, Swiss Federal Institute of Technology (ETHZ), Zurich, Switzerland, for advice on 3-D quantized device simulations in nanoscale.

\section{REFERENCES}

[1] J.-P. Colinge, C.-W. Lee, A. Afzalian, N. D. Akhavan, R. Yan, I. Ferain, P. Razavi, B. O’Neill, A. Blake, M. White, A.-M. Kelleher, B. McCarthy, and R. Murphy, "Nanowire transistors without junctions," Nature Nanotechnol., vol. 5, no. 3, pp. 225-229, Mar. 2010.

[2] J.-P. Colinge, D. Lederer, A. Afzalian, R. Yan, C.-W. Lee, N. D. Akhavan, and W. Xiong, "Properties of accumulation-mode multi-gate field-effect transistors," Jpn. J. Appl. Phys., vol. 48, no. 3, p. 034502, Mar. 2009.

[3] M.-C. M. Lee and M. C. Wu, "Thermal annealing in hydrogen for 3-D profile transformation on silicon-on-insulator and sidewall roughness reduction," J. Microelectromech. Syst., vol. 15, no. 2, pp. 338-343, Apr. 2006.

[4] H. I. Liu, D. K. Biegelsen, N. M. Johnson, F. A. Ponce, and R. F. W. Pease, "Self-limiting oxidation of Si nanowires," J. Vac. Sci. Technol. B, vol. 11, no. 6, pp. 2532-2537, Nov. 1993.

[5] M. Najmzadeh, D. Bouvet, W. Grabinski, and A. M. Ionescu, "Uniaxially tensile strained accumulation-mode gate-all-around $\mathrm{Si}$ nanowire nMOSFETs," in Proc. IEEE DRC, 2011, pp. 145-146.
[6] M. Najmzadeh, D. Bouvet, W. Grabinski, and A. M. Ionescu, "Accumulation-mode GAA Si NW nFET with sub-5 nm cross-section and high uniaxial tensile strain," in Proc. IEEE ESSDERC, 2011, pp. 311-314.

[7] M. Najmzadeh, D. Bouvet, W. Grabinski, and A. M. Ionescu, "Local stressors to accommodate 1.2 to $5.6 \mathrm{GPa}$ uniaxial tensile stress in suspended gate-all-around Si nanowire nMOSFETs by elastic local buckling," in Proc. IEEE ISDRS, 2011, pp. 1-2.

[8] M. Najmzadeh, Y. Tsuchiya, D. Bouvet, W. Grabinski, and A. M. Ionescu, "Multi-gate buckled self-aligned dual Si nanowire MOSFETs on bulk Si for high electron mobility," IEEE Trans. Nanotechnol., vol. 11, no. 5, pp. 902-906, Sep. 2012.

[9] M. Najmzadeh, D. Bouvet, W. Grabinski, J.-M. Sallese, and A. M. Ionescu, "Accumulation-mode gate-all-around Si nanowire nMOSFETs with sub-5 nm cross-section and high uniaxial tensile strain," Solid State Electron., vol. 74, pp. 114-120, Aug. 2012.

[10] J.-P. Colinge, FinFETs and Other Multi-Gate Transistors. New York: Springer-Verlag, 2007.

[11] S. Barraud, M. Berthomé, R. Coquand, M. Cassé, T. Ernst, M. Samson, P. Perreau, K. K. Bourdelle, O. Faynot, and T. Poiroux, "Scaling of trigate junctionless nanowire MOSFET with gate length down to $13 \mathrm{~nm}$," IEEE Electron Device Lett., vol. 33, no. 9, pp. 1225-1227, Sep. 2012.

[12] C.-P. Li, C.-S. Lee, X.-L. Ma, N. Wang, R.-Q. Zhang, and S.-T. Lee, "Growth direction and cross-sectional study of silicon nanowires," Adv. Mater., vol. 15, no. 7/8, pp. 607-609, Apr. 2003.

[13] Y. Wu, Y. Cui, L. Huynh, C. J. Barrelet, D. C. Bell, and C. M. Lieber, "Controlled growth and structures of molecular-scale silicon nanowires," Nano Lett., vol. 4, no. 3, pp. 433-436, Mar. 2004.

[14] K. Tachi, M. Casse, D. Jang, C. Dupre, A. Hubert, N. Vulliet, V. Maffini-Alvaro, C. Vizioz, C. Carabasse, V. Delaye, J. M. Hartmann, G. Ghibaudo, H. Iwai, S. Cristoloveanu, O. Faynot, and T. Ernst, "Relationship between mobility and high-k interface properties in advanced Si and SiGe nanowires," in Proc. IEDM, 2009, pp. 1-4.

[15] M. G. Ancona and G. J. Iafrate, "Quantum correction to the equation of state of an electron gas in a semiconductor," Phys. Rev. B, vol. 39, no. 13, pp. 9536-9540, May 1989.

[16] Sentaurus Device User Guide Version G-2012.06, Synopsys, Mountain View, CA, 2012.

[17] D. B. M. Klaassen, J. W. Slotboom, and H. C. de Graaff, "Unified apparent bandgap narrowing in n- and p-type silicon," Solid State Electron., vol. 35, no. 2, pp. 125-129, Feb. 1992

[18] J.-M. Sallese, N. Chevillon, C. Lallement, B. Iniguez, and F. Pregaldiny, "Charge-based modeling of junctionless double-gate field-effect transistors," IEEE Trans. Electron Devices, vol. 58, no. 8, pp. 2628-2637, Aug. 2011.

[19] F. J. Garcia Ruiz, A. Godoy, F. Gamiz, C. Sampedro, and L. Donetti, "A comprehensive study of the corner effects in pi-gate MOSFETs including quantum effects," IEEE Trans. Electron. Devices, vol. 54, no. 12, pp. 3369-3377, Dec. 2007.

[20] H.-S. Wong, M. H. White, T. J. Krutsick, and R. V. Booth, "Modeling of transconductance degradation and extraction of threshold voltage in thin oxide MOSFETs," Solid State Electron., vol. 30, no. 9, pp. 953-968, Sep. 1987.

[21] J. G. Fossum, J. W. Yang, and V. P. Trivedi, "Suppression of corner effects in triple-gate MOSFETs," IEEE Electron Device Lett., vol. 24, no. 12, pp. 745-747, Dec. 2003.

[22] K. Uchida, J. Koga, R. Ohba, T. Numata, and S. I. Takagi, "Experimental evidences of quantum-mechanical effects on low-field mobility, gate-channel capacitance, threshold voltage of ultrathin body SOI MOSFETs," in IEDM Tech. Dig., 2001, pp. 29.4.1-294.1.4.

[23] H. Majima, H. Ishikuro, and T. Hiramoto, "Experimental evidence for quantum mechanical narrow channel effect in ultra-narrow MOSFETs," IEEE Electron Device Lett., vol. 21, no. 8, pp. 396-398, Aug. 2000.

[24] Y. Yuan, B. Yu, J. Song, and Y. Taur, "An analytic model for threshold voltage shift due to quantum confinement in surrounding gate MOSFETs with anisotropic effective mass," Solid State Electron., vol. 53, no. 2, pp. 140-144, Feb. 2009.

[25] K. E. Moselund, D. Bouvet, L. Tschuor, V. Pott, P. Dainesi, and A. M. Ionescu, "Local volume inversion and corner effects in triangular gate-all-around MOSFETs," in Proc. IEEE ESSDERC, 2006, pp. 359-362.

[26] S. A. Pervez, H. Kim, B.-G. Park, and H. Shin, "Simulation study for suppressing corner effect in a saddle MOSFET for sub-50 nm high density high performance DRAM cell transistor," in Proc. IEEE ISDRS, 2009, pp. $1-2$.

[27] W. Xiong, J. W. Park, and J. P. Colinge, "Corner effect in multiple-gate SOI MOSFETs," in Proc. IEEE Int. SOI Conf., 2003, pp. 111-113. 


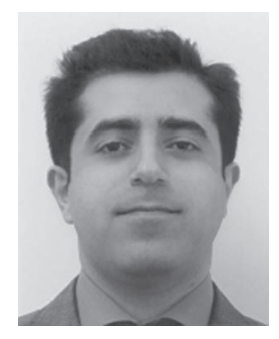

Mohammad Najmzadeh (S'06) is working toward the Ph.D. degree in the Nanoelectronic Devices Lab., Swiss Federal Institute of Technology (EPFL), Lausanne, Switzerland, specialized on modeling, simulation and fabrication of Si nanowire devices.

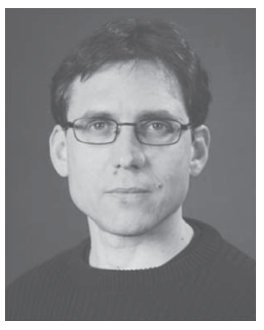

Jean-Michel Sallese is with the Swiss Federal Institute of Technology (EPFL), Lausanne, Switzerland, giving lectures on advanced semiconductor devices and specialized on compact modeling of multi-gate and MEMS devices.

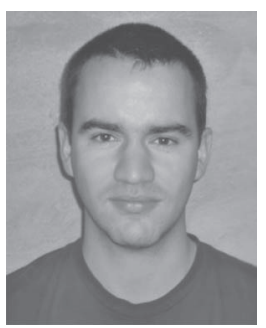

Matthieu Berthomé (S'10) is working toward the Ph.D. degree in the Nanoelectronic Devices Lab., Swiss Federal Institute of Technology (EPFL), Lausanne, Switzerland, specialized on modeling, simulation and fabrication of junctionless NWs.

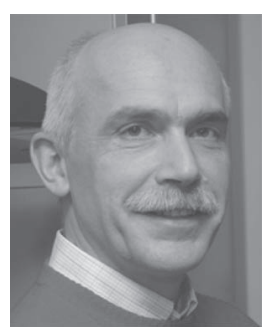

Wladek Grabinski (SM'11) is with the Swiss Federal Institute of Technology (EPFL), Lausanne, Switzerland, specialized on high frequency characterization, compact modeling and device simulation for analog/RF low power applications.

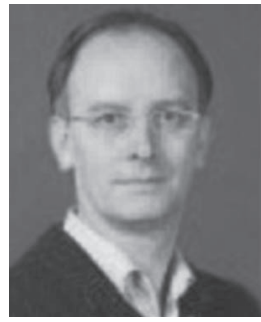

Adrian M. Ionescu (SM'06) is a Full Professor and the Director of the Nanoelectronic Devices Laboratory, Swiss Federal Institute of Technology (EPFL), Lausanne, Switzerland. 